\title{
Heterostructures of bilayer graphene and $h$-BN: Interplay between misalignment, interlayer asymmetry, and trigonal warping
}

\author{
M. Mucha-Kruczyński, ${ }^{1,}{ }^{*}$ J. R. Wallbank, ${ }^{2}$ and V. I. Fal'ko ${ }^{2}$ \\ ${ }^{1}$ Department of Physics, University of Bath, Claverton Down, Bath BA2 7AY, United Kingdom \\ ${ }^{2}$ Department of Physics, Lancaster University, Lancaster LAI 4YB, United Kingdom
}

(Received 1 September 2013; published 18 November 2013)

\begin{abstract}
We study the superlattice minibands produced by the interplay between a moiré pattern induced by a hexagonal BN substrate on a graphene layer and the interlayer coupling in bilayer graphene with Bernal stacking (BLG). We compare moiré miniband features and zero-energy gaps in BLG, where they are affected by the interlayer asymmetry of $\mathrm{BLG} / h$-BN heterostructure and trigonal warping characteristic for electrons in Bernal-stacked bilayers, with those found in monolayer graphene.
\end{abstract}

DOI: 10.1103/PhysRevB.88.205418

PACS number(s): 73.22.Pr, 73.21.Cd, 81.05.ue

The heterostructures of graphene with other hexagonal layered crystals or crystals with hexagonal symmetry facets feature moiré patterns which are the result of incommensurability of the periods of the two two-dimensional lattices, or their misalignment. Since the period of the moiré pattern is longer for a pair of crystals with a closer size of lattice constants and better aligned principal crystallographic axes, the long period moiré superlattices are characteristic for graphene/hexagonal boron nitride $(h-\mathrm{BN})$ heterostructures with a small misalignment angle $\theta$ between the two honeycomb lattices. Such heterostructures have recently been created by transferring graphene onto $h$-BN. ${ }^{1-4}$ The influence of hexagonal moiré patterns on Dirac electrons in monolayer graphene (MLG) has been studied in detail, ${ }^{1-8}$ using both specific microscopic models and phenomenologically. Three possible types of moiré miniband structures on the conduction-/ valence-band sides of graphene's spectrum have emerged from the theories: ${ }^{2-8}$ sometimes, spectra without a distinct separation between the lowest and other minibands; quite exceptionally, the first miniband separated from the next band by a triplet of secondary Dirac points (sDPs) in each of the graphene valleys $K$ and $K^{\prime}$; more generically, a single sDP at the edge of the first miniband in each valley. Also, the signatures of the miniband formation have been observed experimentally in the tunneling density of states ${ }^{2}$ and magnetotransport characteristics ${ }^{3,4}$ in MLG/h-BN heterostructures.

In this paper, we analyze the characteristic moiré miniband features in heterostructures of bilayer graphene (BLG) with highly oriented and almost commensurate, hexagonal crystals, such as $h$-BN, recently created and investigated using magnetotransport measurements by Dean et al. ${ }^{9}$ The analysis below uses and extends the symmetry-based phenomenological approach developed for monolayer graphene. ${ }^{8}$ We find that, in contrast to monolayers, the electronic spectrum of BLG on $h$-BN is most likely to exhibit gaps between the first moiré miniband and the rest of the spectrum (on valenceor conduction-band side, and sometimes in both bands), or have the bands strongly overlapping with each other, whereas sDPs at the miniband edge appear only for exceptionally unique choice of moiré parameters. Also, we find that a gap at the edge between the valence and conduction bands can be opened in BLG by the same moire perturbation that would not open a "zero-energy" gap in MLG. This behavior is prescribed by the substrate creating a moire perturbation only for one layer of BLG, thus breaking the inversion symmetry of the moiré superlattice. The results of our systematic study of the miniband regimes in $\mathrm{BLG} / h$-BN heterostructures are summarized in Fig. 1, where we classify miniband spectra (due to a moiré perturbation characterized by parameters introduced in the description of monolayer graphene on $h$ - $\mathrm{BN}$ ) according to the overlap between the first two minibands on the valence side. Regions of the parameter space corresponding to gapped spectra are painted in red, while those indicating overlapping (nonresolved) bands are left transparent. Differences between the two diagrams, related to different misalignment angles $\theta$, arise from the interplay between the orientation of the supercell Brillouin zone (sBZ) and the skew interlayer hopping in BLG. This interplay, unique to BLG, can help in narrowing down the range of values of microscopic parameters of moiré pattern
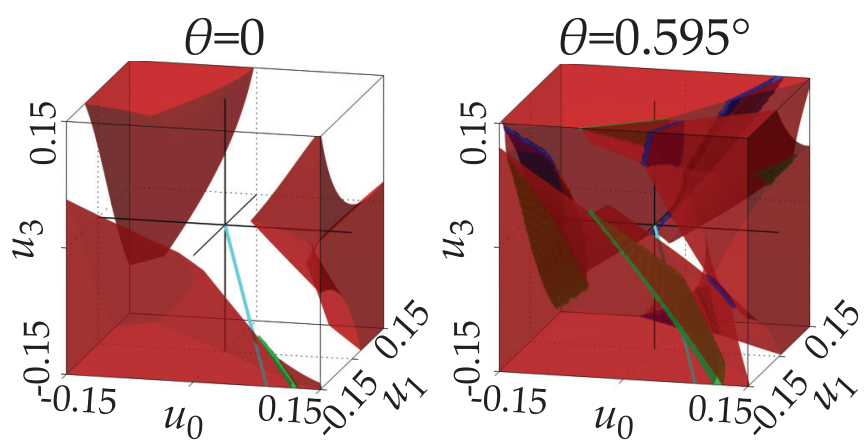

FIG. 1. (Color online) Regimes of miniband spectra over the parameter space used to characterize moiré superlattices in highly oriented BLG on almost commensurate substrate. Here, we use parametrization similar to that introduced ${ }^{8}$ in monolayer studies. In red, we paint regions where the BLG spectrum has a gap separating the first miniband in the valence band from the rest of the spectrum; regions with overlapping (unresolved) bands are transparent and blue/green marks the degenerate conditions for the appearance of isolated secondary Dirac points at the first miniband edge, like in MLG (Refs. 2 and 6-8). The thick cyan lines show directions in the parameter space favored by the point-charge lattice (Ref. 8) and graphene- $h$-BN hopping (Ref. 6) models. A similar parametric plot describing the minibands on the conduction-band side can be obtained by inversion in the $u_{0}-u_{3}$ plane. 
at the graphene $/ h$-BN interface using magnetotransport and capacitance experiments.

The analysis in this paper is performed using a phenomenological approach ${ }^{8}$ which involves the description of the long-range moiré superlattice using a Dirac-type model for graphene electrons, where we include all symmetry-allowed terms in the moiré perturbation applied to one of the two layers in BLG and perform an exhaustive numerical analysis in order to characterize the miniband behavior over a broad range of the parameter space. For BLG placed on top of a substrate with hexagonal symmetry and the lattice constant $a_{\mathrm{S}}=(1+\delta) a$ larger by $\delta$ than that of graphene $[a=2.46 \AA$, and for the case of $h$-BN, $\delta=1.8 \%$ (Ref. 1)], the lattice mismatch, together with a possible misalignment of the two lattices given by the angle $\theta$, lead to a periodic structure which can be described using a set of reciprocal-lattice vectors,

$$
\boldsymbol{b}_{\boldsymbol{n}}=\hat{\boldsymbol{R}}_{n \pi / 3}\left[1-(1+\delta)^{-1} \hat{\boldsymbol{R}}_{\theta}\right]\left(0, \frac{4 \pi}{\sqrt{3} a}\right), \quad n=0,1, \ldots, 6,
$$

where $\hat{\boldsymbol{R}}_{\varphi}$ stands for anticlockwise rotation by angle $\varphi$ and $b=\left|\boldsymbol{b}_{\boldsymbol{n}}\right| \approx \frac{4 \pi}{\sqrt{3} a} \sqrt{\delta^{2}+\theta^{2}}$. Note that this set both rotates by $\phi(\theta)$ and changes its size as a function of $\theta$, Fig. 2. Because of the rapidly decaying nature of the interlayer interaction, we only take into account the influence of $h$-BN on the bottom carbon layer, neglecting any interaction with the top one. Then, following from the MLG investigations, ${ }^{8}$ the electrons in $\mathrm{BLG} / h-\mathrm{BN}$ heterostructure are described by the Hamiltonian

$$
\begin{aligned}
\hat{\boldsymbol{H}} & =\left(\begin{array}{cc}
v \boldsymbol{\sigma} \cdot \boldsymbol{p}+\delta \hat{\boldsymbol{H}}_{\mathrm{sym}}+\delta \hat{\boldsymbol{H}}_{\mathrm{asym}} & \frac{1}{2} \gamma_{1}\left(\tau_{z} \sigma_{x}-i \sigma_{y}\right)+\frac{1}{2} v_{3}\left(\sigma_{x}+i \tau_{z} \sigma_{y}\right)\left(p_{x}+i \tau_{z} p_{y}\right) \\
\frac{1}{2} \gamma_{1}\left(\tau_{z} \sigma_{x}+i \sigma_{y}\right)+\frac{1}{2} v_{3}\left(\sigma_{x}-i \tau_{z} \sigma_{y}\right)\left(p_{x}-i \tau_{z} p_{y}\right) & v \boldsymbol{\sigma} \cdot \boldsymbol{p}
\end{array}\right), \\
\delta \hat{\boldsymbol{H}}_{\mathrm{sym}} & =u_{0} v b f_{1}(\boldsymbol{r})+u_{3} v b f_{2}(\boldsymbol{r}) \sigma_{z} \tau_{z}+u_{1} v\left[\boldsymbol{l}_{z} \times \nabla f_{2}(\boldsymbol{r})\right] \cdot \boldsymbol{\sigma} \tau_{z}+u_{2} v \nabla f_{2}(\boldsymbol{r}) \cdot \boldsymbol{\sigma} \tau_{z}, \\
\delta \hat{\boldsymbol{H}}_{\mathrm{asym}} & =\tilde{u}_{0} v b f_{2}(\boldsymbol{r})+\tilde{u}_{3} v b f_{1}(\boldsymbol{r}) \sigma_{z} \tau_{z}+\tilde{u}_{1} v\left[\boldsymbol{l}_{z} \times \nabla f_{1}(\boldsymbol{r})\right] \cdot \boldsymbol{\sigma} \tau_{z}+\tilde{u}_{2} v \nabla f_{1}(\boldsymbol{r}) \cdot \boldsymbol{\sigma} \tau_{z} \\
f_{1}(\boldsymbol{r}) & =\sum_{n} e^{i \boldsymbol{b}_{n} \cdot \boldsymbol{r}}, \quad f_{2}(\boldsymbol{r})=i \sum_{n}(-1)^{n} e^{i \boldsymbol{b}_{\boldsymbol{n}} \cdot \boldsymbol{r}},
\end{aligned}
$$

written in the basis of the Bloch states on sublattices $\left\{\phi\left(A_{1}\right), \phi\left(B_{1}\right), \phi\left(A_{2}\right), \phi\left(B_{2}\right)\right\}$ in the $K$ valley and $\left\{\phi\left(B_{1}\right),-\right.$ $\left.\phi\left(A_{1}\right), \phi\left(B_{2}\right),-\phi\left(A_{2}\right)\right\}$ in $K^{\prime}$, where indices $1 / 2$ mark the bottom/top layers and the substrate directly acts on the electrons in the bottom layer. We also use $\hbar=1$ and employ two sets of Pauli matrices $\sigma_{i}, \sigma=\left(\sigma_{x}, \sigma_{y}\right)$, and $\tau_{i}$, acting in the sublattice and valley space, respectively.

The intralayer Dirac-like terms $v \boldsymbol{\sigma} \cdot \boldsymbol{p}$ on the diagonal and interlayer off-diagonal terms on the right-hand side of Eq. (1) follow from the tight-binding description of BLG, ${ }^{10}$ with $v=10^{6} \mathrm{~m} / \mathrm{s}$ resulting from the interlayer hopping in graphene. Also, $\gamma_{1}=0.38 \mathrm{eV}$ (Ref. 11) characterizes the "vertical" interlayer hopping between the closest neighbors and $v_{3} \approx 0.12 v$ (Refs. 10-12) describes the trigonal warping of BLG bands, resulting from the skew next-neighbor interlayer hopping. This results in the four-band BLG model, with two bands degenerate at the points $K$ and $K^{\prime}$, and two bands split away by $\pm \gamma_{1}$.

The moiré perturbation, scaled using energy scale $v b$ and parametrized using dimensionless $\left\{u_{i}, \tilde{u}_{i}\right\}, i=0,1,2,3{ }^{8}$ captures the effect of the substrate on BLG through its coupling with carbon orbitals in the closest, bottom layer only. Following the approach used in the monolayer study, ${ }^{8}$ we separate the moiré perturbation due to $h$-BN into inversion-symmetric and asymmetric terms, $\delta \hat{\boldsymbol{H}}_{\text {sym }}$ and $\delta \hat{\boldsymbol{H}}_{\text {asym, }}$, respectively. By considering the limiting case in which electrons in graphene are dominantly affected by only one of the two atoms in the $h$-BN unit cell, we argue that $\left|\tilde{u}_{i}\right| \ll\left|u_{i}\right|$ and neglect $\delta \hat{\boldsymbol{H}}_{\text {asym }}$ in all further discussions of the band structures, except the analysis of a gap opened at zero energy, where it is necessary in order to compare to the situation in monolayer graphene. The remaining parameters, $u_{0,1,2,3}$, can be associated with the following characteristics of the moiré pattern: parameter $u_{0}$ characterizes the magnitude of a smooth electrostatic potential, $u_{3}$ captures the local asymmetry between the $A_{1}$ and $B_{1}$ sublattices, and $u_{1}$ and $u_{2}$ introduce modulation of the in-plane hops for the electrons traveling within the bottom layer. As opposed to the monolayer case, ${ }^{8}$ in BLG the rotorless $u_{2}$ term cannot be completely gauged away. However, it vanishes for zero misalignment angle, and its effect on the band structure is generated via the interplay with the trigonal warping term: therefore, it is small and can be neglected. Note that, since we scale all energies by $v b \approx v \frac{4 \pi}{\sqrt{3} a} \sqrt{\delta^{2}+\theta^{2}}$, the size of dimensionless parameters $u_{i}$ in Eq. (1) would be larger for smaller angle $\theta$ for the same pair of BLG and a substrate.

Due to the time-inversion symmetry, described by the operation $^{13,14}$

$$
\hat{\boldsymbol{H}}(\boldsymbol{p})=\sigma_{y} \tau_{y}\left[\hat{\boldsymbol{H}}^{*}(-\boldsymbol{p})\right] \sigma_{y} \tau_{y},
$$

electronic spectra in the two valleys are related, $\epsilon_{\boldsymbol{K}+\boldsymbol{p}}=\epsilon_{\boldsymbol{K}^{\prime}-\boldsymbol{p}}$, so that we only discuss electronic spectra in one valley, e.g.,

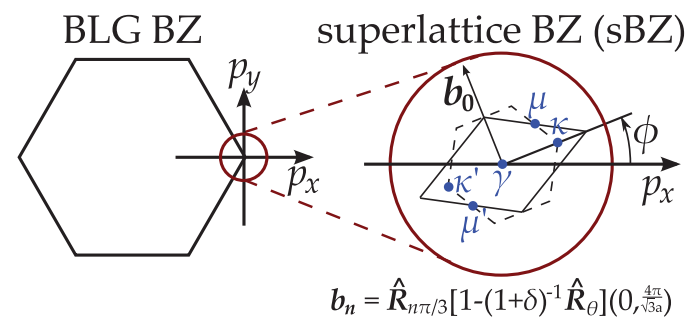

FIG. 2. (Color online) Hexagonal Brillouin zone (BZ) of BLG with the valley coordinate system $p_{x}, p_{y}$ and the electronic bands in the vicinity of the $K$ valley, together with the zoom in on the supercell Brillouin zone (sBZ) where we mark its own symmetry points $\kappa$ and $\mu$. 
$K$. We find that in contrast to unperturbed BLG, the spectrum resulting from a generic choice of parameters in Hamiltonian (1) is not electron-hole symmetric, but obeys the following relation:

$$
\epsilon_{\boldsymbol{K}+\boldsymbol{p}}^{u_{0}, u_{1}, u_{3}}=-\epsilon_{\boldsymbol{K}+\boldsymbol{p}}^{-u_{0}, u_{1},-u_{3}},
$$

which folds the parameter space $\left(u_{0}, u_{1}, u_{3}\right)$ to be explored.

For the sake of a systematic comparison with the lowenergy "two-band" model of free-standing BLG, ${ }^{10}$ applicable at the energy scale $\epsilon \ll \gamma_{1}$, we use a Schrieffer-Wolff transformation ${ }^{15}$ and project the four-band Hamiltonian onto the low-energy bands, reducing it to an effective two-band Hamiltonian,

$$
\begin{aligned}
\hat{\boldsymbol{H}}_{\mathrm{eff}} \approx & -\frac{v^{2}}{\gamma_{1}}\left[\left(p_{x}^{2}-p_{y}^{2}\right) \sigma_{x}+2 p_{x} p_{y} \sigma_{y}\right] \tau_{z}+v_{3}(\boldsymbol{\sigma} \cdot \boldsymbol{p})^{T} \\
& +\frac{v b}{2} g_{+}(\boldsymbol{r})\left(1+\sigma_{z} \tau_{z}\right)+\frac{v^{3} b}{2 \gamma_{1}^{2}}\left(p_{x}-i \sigma_{z} p_{y}\right) g_{-}(\boldsymbol{r}) \\
& \times\left(p_{x}+i \sigma_{z} p_{y}\right)\left(1-\sigma_{z} \tau_{z}\right) \\
& +\frac{v^{2} b}{2 \gamma_{1}}\left[\left(p_{x}+i p_{y} \tau_{z}\right) g(\boldsymbol{r})\left(\sigma_{x}-i \sigma_{y} \tau_{z}\right)\right. \\
& \left.+g^{*}(\boldsymbol{r})\left(p_{x}-i p_{y} \tau_{z}\right)\left(\sigma_{x}+i \sigma_{y} \tau_{z}\right)\right]
\end{aligned}
$$

where

$$
\begin{aligned}
g_{ \pm}(\boldsymbol{r})= & \left(u_{0} \pm \tilde{u}_{3}\right) f_{1}(\boldsymbol{r}) \pm\left(u_{3} \pm \tilde{u}_{0}\right) f_{2}(\boldsymbol{r}), \\
g(\boldsymbol{r})= & \sum_{n} e^{i \boldsymbol{b}_{\boldsymbol{n}} \cdot \boldsymbol{r}}\left(\hat{b}_{n}^{x}+i \hat{b}_{n}^{y} \tau_{z}\right) \\
& \times\left[(-1)^{n}\left(u_{2}+i u_{1} \tau_{z}\right)+\left(\tilde{u}_{1} \tau_{z}-i \tilde{u}_{2}\right)\right], \\
\boldsymbol{b}_{\boldsymbol{n}}= & \left(\hat{b}_{n}^{x}, \hat{b}_{n}^{y}\right) b .
\end{aligned}
$$

The applicability of the simplified Hamiltonian to the description of, at least, the first moiré miniband in the BLG spectrum requires that $\gamma_{1} \gtrsim 2 v b$. For a perfectly aligned BLG/ $h$-BN heterosystem, we estimate that $\frac{\gamma_{1}}{v b} \approx \frac{\sqrt{3}(1+\delta)}{4 \pi} \frac{a \gamma_{1}}{v}=$ 1.107, which suggests that a quantitative description of moiré minibands in $\mathrm{BLG} / h-\mathrm{BN}$ requires the use of the four-band Hamiltonian, Eq. (1).

Little is known about the exact values of each of the perturbation parameters $u_{i}$. Due to the size of the moiré and the importance of van der Waals interaction in graphene/ $h$-BN heterostructures, resources needed for supercell-size $a b$ initio calculations are prohibitive. At the same time, even for the more studied system of MLG/ $h-\mathrm{BN}$, experimental data obtained so far also do not allow for clear determination of the perturbation. ${ }^{3,4}$ Hence, in our numerical modeling of spectra, we broadly cover the $\left(u_{0}, u_{1}, u_{3}\right)$ parameter space, Fig. 1. Examples of characteristic moiré miniband spectra on the valence-band side in $\mathrm{BLG} / h-\mathrm{BN}$ are shown in Fig. 3: (a) overlapping minibands characteristic for the transparent part of the parameter space in Fig. 1; (b) and (c) gapped spectrum at the edge of the first moiré miniband characteristic for the red-painted part of the parameter space in Fig. 1. Also shown in Figs. 3(b) and 3(c) are the corresponding densities of states, with a global gap in the valence band.

Spectra in Figs. 3(a) and 3(c) both correspond to the same choice of perturbation, $u_{0}=u_{1}=0, u_{3}=0.15$, but a different misalignment angle $\theta$, which is enough to cause the opening of a band gap between the first and second minibands on (a) $\theta=0, v b=0.903 \gamma_{1}, u_{3}=0.15, u_{0}=u_{1}=0$
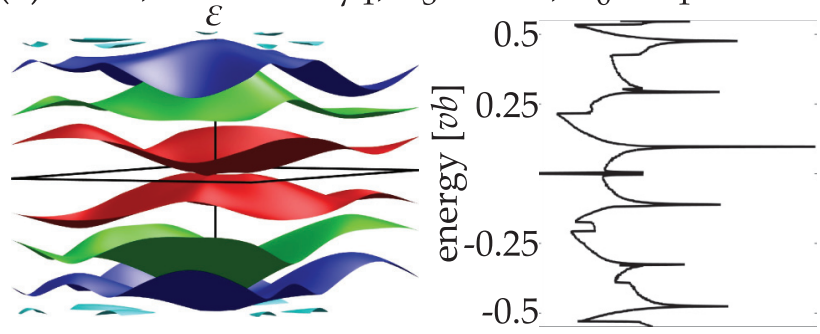

density of states [arb.units]

(b) $\theta=0, v b=0.903 \gamma_{1}, u_{0}=0.15, u_{1}=u_{3}=0$
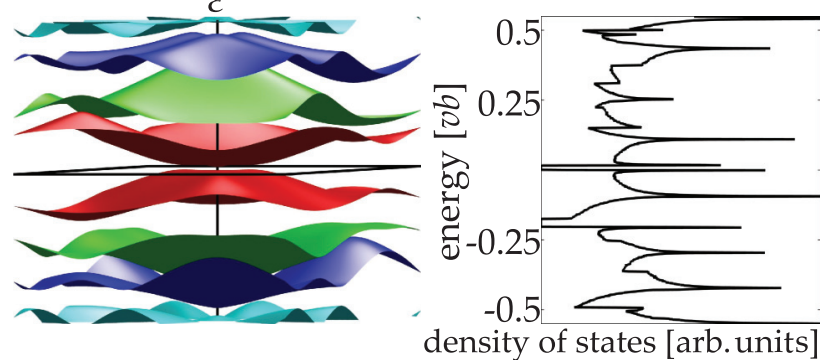

(c) $\theta=0.595^{\circ}, v b=1.053 \gamma_{1}, u_{3}=0.15, u_{0}=u_{1}=0$


FIG. 3. (Color online) (a)-(c) Moiré miniband spectra (drawn within the rhombic sBZ) and density of states (DoS) portraying two characteristic behaviors of the miniband spectrum as determined in Fig. 1.

the valence side. This is because of the trigonal warping of the unperturbed BLG spectrum, which shifts the energy $\epsilon_{p}^{0}$ of a momentum state $\boldsymbol{p}=\left(p_{x}, p_{y}\right)$ by $\epsilon_{\text {warp }} \approx-s v_{3} p \cos 3 \varphi$, where $\varphi=\arctan \frac{p_{y}}{p_{x}}, p=\sqrt{p_{x}^{2}+p_{y}^{2}}$ and $s=1 \quad(s=-1)$ denotes the conduction (valence) band. For $\theta=\phi=0$, this results, for example, in the $\kappa\left(\kappa^{\prime}\right)$ point in the valence band shifted up (down) in energy, like in Fig. 3(a). However, misalignment angle $\theta=0.595^{\circ}$ leads to $\mathrm{sBZ}$ rotation by $\phi=-30^{\circ}$ and the trigonal warping correction at the points $\kappa$ and $\kappa^{\prime}$ of the rotated sBZ vanishes, as seen in Fig. 3(c). Such a strong dependence of the miniband spectrum on the misalignment angle is special to BLG, because in MLG trigonal warping corrections are much weaker. Consequently, experimental investigation of the BLG miniband spectra for several misalignment angles (determined from the moiré geometry) may yield new information about the nature of the perturbation felt by graphene electrons due to $h$-BN. For example, two of the models suggested for the MLG/h-BN heterostructure, the graphene- $h$-BN hopping model ${ }^{6}$ and the point-charge lattice model, ${ }^{8}$ yield the same form of the $u_{i}$ 


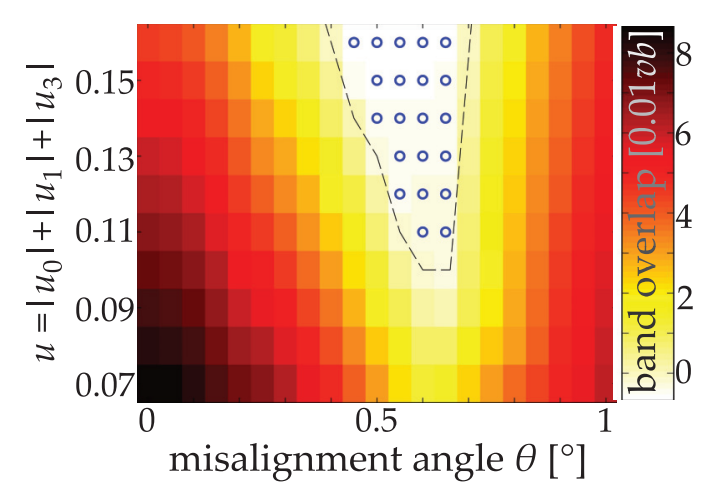

FIG. 4. (Color online) The size of the overlap between the first and second miniband on the valence side as a function of the perturbation magnitude $u=\sum_{i}\left|u_{i}\right|$ and the misalignment angle $\theta$, assuming that the perturbation parameters are described by relations in Eq. (3). Negative values for the overlap indicate the parameter range where the spectrum is gapped-above the dashed line, and points marked with blue circles correspond to the spectra for which the global gap is direct at $\kappa^{\prime}$. In contrast, on the conduction side, the minibands always overlap.

coefficients as a function of the misalignment angle $\theta$,

$$
u_{0}=\frac{1}{2} \tilde{v}, \quad u_{1}=-\frac{\delta}{\sqrt{\delta^{2}+\theta^{2}}} \tilde{v}, \quad u_{3}=-\frac{\sqrt{3}}{2} \tilde{v}
$$

( $\tilde{v}>0$ is a strength of the perturbation), corresponding to a single line for each misalignment angle $\theta$, as shown with bold cyan lines in Fig. 1 for $\theta=0$ and $\theta=0.595^{\circ}$. It is interesting to note that in the diagram for $\theta=0.595^{\circ}$, this line passes close behind the green region, for which a secondary Dirac point at $\kappa^{\prime}=\left(-\frac{1}{\sqrt{3}} b, 0\right)$, not obstructed on the energy scale by other spectral features, separates the first and the second miniband on the valence side. In Fig. 4, we show a detailed study of the overlap between the first and second miniband on the valence side as a function of the misalignment angle $\theta$ and perturbation with parameters $u_{i}$ related to each other by Eq. (3) and a magnitude $u=\sum_{i}\left|u_{i}\right|$. Due to trigonal warping, for $\theta \approx 0.6^{\circ}$ and big enough $u$, the miniband spectra display a small band gap, as indicated by the brightest part of the figure corresponding to negative values of the overlap. For points marked with a blue circle, this is a direct gap at the $\kappa^{\prime}$ point. In contrast, on the conduction side the minibands always overlap. Note that even a small change of misalignment angle leads to a large rotation of moiré pattern and a different involvement of trigonal warping due to skew interlayer coupling in BLG. The corresponding evolution of BLG moiré spectra with increasing misalignment angle is illustrated in Fig. 5. For $\theta=0$, left column in Fig. 5, trigonal warping affects $\kappa$ and $\kappa^{\prime}$ in the opposite fashion (shown in the inset), what obscures the sDP at $\kappa^{\prime}$. However, the sDP becomes isolated on the energy scale for $\theta \approx 0.6^{\circ}$, center column in Fig. 5. It disappears again as the misalignment angle is increased further, with the asymmetry between $\kappa$ and $\kappa^{\prime}$ reaching the maximum for $\theta=1.786^{\circ}$ when the $\mathrm{sBZ}$ is rotated by $60^{\circ}$, right column in Fig. $5 .^{16}$

One can notice that in the majority of the spectra presented in Figs. 3 and 5, and for generic moiré perturbation, a gap, $\Delta$, is opened at the "neutrality point" between the conduction and
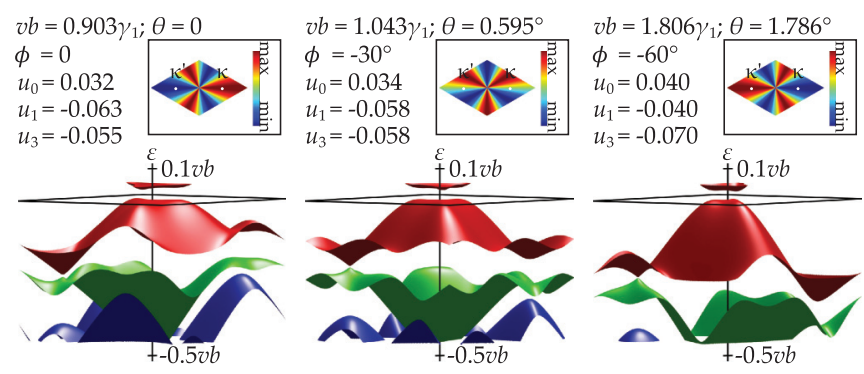

FIG. 5. (Color online) Moiré miniband spectra (drawn within the rhombic sBZ) illustrating the role of trigonal warping in determining whether the first and second minibands on the valence side are overlapping or gapped at a secondary Dirac point. For these examples, we assumed that relations in Eq. (3) hold and set $u=\sum_{i}\left|u_{i}\right|=0.15$. The insets show the angular dependence, $\cos 3 \phi$, of the trigonal warping for the valence band within the (rotated) sBZ.

valence bands, in contrast to monolayer graphene, where such gap,

$$
\Delta^{\mathrm{MLG}}=24 v b\left|u_{1} \tilde{u}_{0}+u_{0} \tilde{u}_{1}\right|
$$

appears only when the moiré pattern contains an inversion asymmetric perturbation. In the monolayer, this is accompanied by an overall shift, $12 v b\left(u_{1} u_{3}+\tilde{u}_{1} \tilde{u}_{3}\right)$, resulting in the edges of the conduction/valence band at $\epsilon_{ \pm}^{\mathrm{MLG}}=$ $12 v b\left(u_{1} u_{3}+\tilde{u}_{1} \tilde{u}_{3}\right) \pm 12 v b\left(u_{1} \tilde{u}_{0}+u_{0} \tilde{u}_{1}\right)$. In BLG, one of the two degenerate zero-energy states belongs to the bottom and one to the top layer. Because the top layer is unaffected by the perturbation, the corresponding state stays at zero energy, while the other one is shifted by $\epsilon_{+}^{\mathrm{MLG}}$, just like one of the states in MLG. As a result, the bilayer gap is nonzero even if the moiré perturbation is inversion symmetric,

$$
\Delta^{\mathrm{BLG}} \approx 12 v b\left|u_{1}\left(u_{3}+\tilde{u}_{0}\right)+\tilde{u}_{1}\left(u_{0}+\tilde{u}_{3}\right)\right|,
$$

as a consequence of the $h$-BN substrate breaking the equivalence of the two layers and, hence, the inversion symmetry. For BLG sandwiched between two $h$-BN layers, the asymmetry will be still present, if the misalignment angles between graphene and top/bottom $h$-BN layers are different.

In summary, we showed that the interplay between interlayer coupling (including skew hopping between layers leading to the trigonal warping effect in BLG) in bilayer graphene and breaking of layer symmetry by the substrate play an important role in determining the miniband spectrum of BLG $/ h$-BN heterostructures. As opposed to MLG in which a gap at the Dirac point is open only for an inversion-asymmetric moiré perturbation, in BLG a "zero-energy" gap is open even for an inversion-symmetric perturbation, as a direct consequence of interlayer asymmetry caused by the substrate.

This work has been supported by the ERC Advanced Grant Graphene and Beyond, EU Graphene Flagship, EU STREP ConceptGraphene, CDT NOWNano, Royal Society Wolfson Research Merit Award, and EPSRC Science and Innovation Award. 
*m.mucha-kruczynski@bath.ac.uk

${ }^{1}$ J. Xue, J. Sanchez-Yamagishi, D. Bulmash, P. Jacquod, A. Deshpande, K. Watanabe, T. Taniguchi, P. Jarillo-Herrero, and B. J. LeRoy, Nat. Mater. 10, 282 (2011).

${ }^{2}$ M. Yankowitz, J. Xue, D. Cormode, J. D. Sanchez-Yamagishi, K. Watanabe, T. Taniguchi, P. Jarillo-Herrero, P. Jacquod, and B. J. LeRoy, Nat. Phys. 8, 382 (2012).

${ }^{3}$ L. A. Ponomarenko, R. V. Gorbachev, D. C. Elias, G. L. Yu, A. S. Mayorov, J. Wallbank, M. Mucha-Kruczynski, A. Patel, B. A. Piot, M. Potemski, I. V. Grigorieva, K. S. Novoselov, F. Guinea, V. I. Fal'ko, and A. K. Geim, Nature (London) 497, 594 (2013).

${ }^{4}$ B. Hunt, J. D. Sanchez-Yamagishi, A. F. Young, M. Yankowitz, B. J. LeRoy, K. Watanabe, T. Taniguchi, P. Moon, M. Koshino, P. Jarillo-Herrero, and R. C. Ashoori, Science 340, 1427 (2013).

${ }^{5}$ B. Sachs, T. O. Wehling, M. I. Katsnelson, and A. I. Lichtenstein, Phys. Rev. B 84, 195414 (2011).

${ }^{6}$ M. Kindermann, B. Uchoa, and D. L. Miller, Phys. Rev. B 86, 115415 (2012).

${ }^{7}$ C. Ortix, L. Yang, and J. van den Brink, Phys. Rev. B 86, 081405 (2012).

${ }^{8}$ J. R. Wallbank, A. A. Patel, M. Mucha-Kruczynski, A. K. Geim, and V. I. Fal'ko, Phys. Rev. B 87, 245408 (2013).
${ }^{9}$ C. R. Dean, L. Wang, P. Maher, C. Forsythe, F. Ghahari, Y. Gao, J. Katoch, M. Ishigami, P. Moon, M. Koshino, T. Taniguchi, K. Watanabe, K. L. Shepard, J. Hone, and P. Kim, Nature (London) 497, 598 (2013).

${ }^{10}$ E. McCann and V. I. Fal'ko, Phys. Rev. Lett. 96, 086805 (2006).

${ }^{11}$ A. B. Kuzmenko, I. Crassee, D. van der Marel, P. Blake, and K. S. Novoselov, Phys. Rev. B 80, 165406 (2009).

${ }^{12}$ K. Kechedzhi, V. I. Fal'ko, E. McCann, and B. L. Altshuler, Phys. Rev. Lett. 98, 176806 (2007).

${ }^{13}$ Y. Lemonik, I. L. Aleiner, and V. I. Fal'ko, Phys. Rev. B 85, 245451 (2012).

${ }^{14}$ I. L. Aleiner and K. B. Efetov, Phys. Rev. Lett. 97, 236801 (2006). ${ }^{15}$ J. R. Schrieffer and P. A. Wolff, Phys. Rev. 149, 491 (1966).

${ }^{16}$ We tested all of our above conclusions for a "single-side gate" geometry, where a large charge density required to fill the first miniband (four electrons per moiré supercell) is induced by a single gate, which induces additional interlayer asymmetry (Refs. 17 and 18), and found that its presence has almost no effect on the type of the miniband spectrum at the edge of the first miniband (gapped or overlapping minibands).

${ }^{17}$ E. McCann, Phys. Rev. B 74, 161403 (2006).

${ }^{18}$ M. Mucha-Kruczynski, E. McCann, and V. I. Fal'ko, Solid State Commun. 149, 1111 (2009). 